\title{
Fabrication of gold nanoparticles/graphene oxide films with surface-enhanced Raman scattering activity by a simple electrostatic self-assembly method
}

\author{
Yi Li, Juan Yang*, Yazhou Zhou, Nan Zhao, Weiwei Zeng, Wenwei Wang \\ (School of Materials Science and Engineering, Jiangsu University, Zhenjiang, Jiangsu, 212013, China) \\ *Corresponding author, email: yangjuan6347@ujs.edu.cn
}

\begin{abstract}
The paper reports a simple electrostatic self-assembly method to fabricate the $\mathrm{Au}$ nanoparticles/graphene oxide (Au NPs@GO) films which present superior SERS activity. The Au NPs@GO films with different layers construction and surface compositions (with Au NPs or GO as the outer layer) are obtained simply by changing the self-assembly cycles. Results indicate that the film with two assembly cycles (F-2 film) exhibits the best SERS activity towards R6G molecules compared with that of one assembly cycle (F-1 film) and three assembly cycles (F-3 film) due to the high dosage of the absorbed Au NPs exposed on the surface of the film. Meanwhile, the Raman enhancement effect of the F-2 film with the outer layer of Au NPs is better than that of the outer layer covering with GO sheets (F-2-GO) possibly owing to the optimal synergetic effect of $\mathrm{Au}$ nanoparticles and GO. Furthermore, our fabricated Au NPs@GO films present favorable reproducibility and high stability, which are significant for the practical application of these SERS substrates.
\end{abstract}

Key words: Electrostatic self-assembly method; Au NPs@GO films; surface-enhanced Raman scattering; R6G molecules 


\section{Introduction}

As an effective and powerful analytical technique, surface-enhanced Raman scattering (SERS) is generally used for ultrasensitive chemical or biochemical analysis [1-3]. The traditional SERS substrates are usually the noble metal nanoparticles ( $\mathrm{Au}, \mathrm{Ag}$ et al.) [4-7], and the enhancement effect of which is the huge electromagnetic mechanism (EM) depending on the local electromagnetic field. Recently, graphene and its derivative of graphene oxide (GO) [8-10] were found possessing the ability to enhance the Raman signals effectively through the so-called chemical mechanism (CM) based on charge transfer between the detected molecules and the SERS substrate [8]. Therefore, it is necessary to fabricate the noble metal nanoparticles/GO nanocomposites to combine the two different enhancement mechanisms and reach a desirable synergistic effect.

It is well known that there are numerous attempts to fabricate graphene and GO with noble metal nanoparticles $[11,12]$. Generally, the in suit reduction method and self-assembly approach are employed to synthesize these kinds of nanocomposites. For instance, Iliut et al. [13] have investigated that the Au nanoparticles and reduced graphene oxide (Au NPs/rGO) nanocomposites were fabricated by an in suit growth method and stabilized by the surfactant of polyvinylpyrrolidone (PVP). Fan et al. [14] reported that GO and the shape-controlled Ag nanoparticles were prepared on the surface of 3-aminopropyltriethoxysilane (APTES)-modified silicon substrates to synthesize silver nanoparticles/graphene oxide films (Ag NPs@GO) by an electrostatic self-assembly method. However, the dosage of Ag nanoparticles on GO sheets was much low, which would influence the sensitivity and reproducibility of the nanocomposite films. Liu et al. [15] reported that Ag NPs and GO were assembled on the glass substrate to fabricate Ag NPs@GO films, but they only studied the film with one assembly cycle, which has ignored the 
influence of assembly cycles on the enhancement of SERS signals. To the best of our knowledge, the assembly cycles are of paramount importance to the nanoparticles dosage, which would affect the formation of "hot spots" and SERS activities of the composite films [16-19]. However, few trials for constructing the Au NPs@GO films with different assembly cycles to reach an optimum enhancement effect have ever been reported. Furthermore, Ling et al. [8] reported that the SERS sensitivity of graphene films would decrease with the increasing numbers of graphene layers. Therefore, fabricating a kind of Au NPs@GO film with few GO layers to investigate the relationship between the assembly cycles and the intensity of Raman signals may be an interesting work.

Our previously electrostatic self-assembly strategy has been adopted to fabricate Au NPs@GO films [20] successfully. Herein, in order to study how the assembly cycles of Au NPs@GO film will influence the SERS signal intensity, this kind of electrostatic self-assembly method is also employed using Au nanoparticles with positive charges and GO sheets with negative charges. The prepared Au NPs@GO films with different layers construction and surface compositions exhibit diverse SERS enhancement effects against Rhodamine 6G (R6G) molecules.

\section{Experimental}

\subsection{Preparation of Au NPs colloid with positive charges}

The Au NPs colloid was prepared according to a seed-mediated growth method [21]. In a typical procedure, ice-cold sodium borohydride $\left(\mathrm{NaBH}_{4}\right)(10 \mathrm{mM}, 600 \mu \mathrm{L})$ was injected into a mixture containing Chloroauric acid $\left(\mathrm{HAuCl}_{4}\right)(10 \mathrm{mM}, 300 \mu \mathrm{L})$ and Cetyltrimethyl Ammonium Bromide (CTAB) $(100 \mathrm{mM}, 7.5 \mathrm{~mL})$ and stirred for two hours at ambient temperature. After diluted ten times with deionized water, the seed solution can be prepared. In order to get the growth 
solution, $200 \mu \mathrm{L}$ of the upper seed solution was mixed to a mixture (containing $15 \mathrm{~mL}$ deionized (DI) water; $3 \mathrm{~mL}, 100 \mathrm{mM} \mathrm{CTAB} ; 400 \mu \mathrm{L}, 10 \mathrm{mM} \mathrm{HAuCl}_{4}$ and $2 \mathrm{~mL}, 100 \mathrm{mM}$ ascorbic acid) filled in a vial. Subsequently, the vial was inversed rapidly for ten seconds and moved into an oven with $30{ }^{\circ} \mathrm{C}$ for 10 hours. The final Au NPs colloid was obtained by washing the as-mentioned growth solution with DI water for 5 times.

\subsection{Synthesis of the Au NPs@GO film}

GO was synthesized using an improved Hummers method [22]. The pretreated quartz substrate is on the basis of our former study [20]. Firstly, the as-mentioned quartz substrate was immerged in GO aqueous solution for $20 \mathrm{~min}$. Then, in order to eliminate the unstable GO nanosheets, the substrate was washed by DI water, and dried by flowing air using aurilave. After that, the substrate absorbed with GO sheets then was immerged in Au NPs colloid for else 20 minutes, and washed as well as dried in the same procedure. Multilayer Au NPs@GO films were fabricated through duplicating these assembly cycles. Films with 1, 2 and 3 assembly cycles were labeled as F-n (n is the cycle index with the outer layer of Au NPs). Particularly, the film of two assembly cycles covered by GO layers on the surface was marked as F-2-GO. Additionally, for contrast, the pure GO film and pure Au NPs were also prepared.

\subsection{Characterization}

UV-6100 spectrophotometer was used to characterize the optical property of Au NPs colloid. X-ray diffraction (XRD) measurements were carried out using a PW1700 diffractometer with $\mathrm{Cu}$ $\mathrm{K} \alpha_{1}$ radiation $(\lambda=1.5405 \AA)$ to investigate the crystal structure of Au NPs. The morphology of Au NPs was characterized using a JEM-2100 transmission electron microscope (TEM) operating at 200 $\mathrm{kV}$. The zeta-potential was read on a Nano ZS90 Zetasizer system. Atomic force microscopy (AFM) $(0.803 \mathrm{~Hz})$ and a JEOL 6460 field emission scanning electron microscope (FE-SEM) were 
employed to survey the surface morphologies of the Au NPs@GO films. Raman spectroscopy was tested on a Senterra R200-L Raman system at $5 \mathrm{~mW}$ with the laser excitation of $532 \mathrm{~nm}$, which is close to the surface plasmon absorption $(560 \mathrm{~nm})$ of Au NPs (in Fig. 1a).

\subsection{Fabrication of SERS detected sample}

Two main procedures are included to prepare the SERS measurement samples. Typically, the Au NPs@GO films were firstly immersed in R6G aqueous solution overnight and rinsed by DI water for twice aiming in removing the untight molecules. Another procedure is to make the films dry by flowing air using aurilave. For each measurement, the acquisition time was ten seconds and three different measurement regions were detected to guarantee the facticity of every single sample.

\section{Results and discussion}

As is well-known that metal nanoparticles surrounded with quaternary ammonium surfactants can be dispersed well in polar media [23]. Herein, we fabricated the Au NPs colloid which was dispersed in DI water by a seed-mediated growth method using CTAB as the surfactant. The surface plasmon (a quantum of plasma oscillation) absorption (in Fig. 1a) of the synthesized Au NPs is characterized by UV-vis, and the typical absorbance peak of $\mathrm{Au}$ is at $\sim 560 \mathrm{~nm}$ due to the transverse electronic oscillation. The phases of the prepared Au NPs were characterized using XRD (in Fig. 1b). It can be seen clearly that the typical peaks at $38.1^{\circ}(111), 44.6^{\circ}(200), 64.1^{\circ}(220), 77.8^{\circ}(311)$ and $82.3^{\circ}$ (222) are attributed to a face centered cubic bulk Au [13]. It is well known that the enhancement of SERS signals is influenced by the morphology and particle size of the nanoparticles. Previous research [24] indicated that the optimal size of metal NPs used for SERS detection was about $100 \mathrm{~nm}$. As the size was under $100 \mathrm{~nm}$, the SERS enhancement would increase with the particle size increasing. Fan et al. [14] reported that the emergence of multiple incisive 
tips/edges and large sizes of metal nanoparticles had efficient light scatters which are meaningful in improving the SERS enhancement. Therefore, the as-fabricated Au NPs with the satisfactory average diameter of $\sim 69 \mathrm{~nm}$ (in Fig. 1d) and multiple sharp tips/edges morphology (in Fig. 1c) present the advantage in preparation of SERS substrates. Furthermore, the color of the Au NPs aqueous solution (in Fig. 1d) is red, indicating that the Au colloid has been synthesized successfully.

Table 1 The zeta-potential of the GO aqueous solution $\left(0.1 \mathrm{mg} \mathrm{mL}^{-1}\right), \mathrm{CTAB}$ and Au NPs colloid.

\begin{tabular}{cccc}
\hline Sample & GO & CTAB & Au NPs \\
\hline Zeta-potential $(\mathrm{mV})$ & -30.8 & +56.0 & +43.0 \\
\hline
\end{tabular}

The zeta-potential measurement was used for characterizing the electrostatic interaction between GO and Au NPs, and the results were shown in Table 1. The average zeta-potential for GO is highly negative $(-30.8 \mathrm{~mW})$ due to the oxygen-rich functional groups. In our experiment, Au NPs was surrounded by CTAB to make the particles possess positive charges $(+43.0 \mathrm{~mW})$, which can be dispersed well in colloidal solution steadily due to the rejection effect between the same charges. The zeta-potential results illustrate that our designed electrostatic self-assembly process to prepare Au NPs@GO films can be obtained using those above-mentioned GO dispersion and Au NPs colloid.

Fig. 2 is the preparation schematics of the Au NPs@GO film. It can be seen that the pretreated quartz substrate with positive charges was immerged in GO dispersion for $20 \mathrm{~min}$ and a few of GO sheets were deposited onto the substrate surface. Next, this quartz substrate covered with GO sheets was then immerged in Au NPs colloid depositing for additional 20 min. The Au NPs with positive charges are easily deposited onto the substrate by electrostatic attraction. Finally, the Au NPs@GO films with different layers construction and surface compositions can be obtained by simply changing the assembly cycles. 
The Fan's report [14] showed that the dosage of Ag NPs on GO sheets was much low and needed further improving, because it cannot guarantee the reproducibility of the SERS substrate. Previous study [20] indicated that such self-assembly method was a versatile strategy to prepare GO films or NPs decorated GO films with controllable microstructure. Herein, we fabricated the Au NPs@GO films by controlling the self-assembly cycles for the purpose of obtaining the SERS substrate with superior SERS performance and reproducibility. As shown in the AFM image of Fig. 3a, the GO sheets with some folds can be seen apparently on the quartz substrate after deposition in GO suspension. The actual deposition process may be complex which is not only single layer adsorption, but also few layers adsorption. And AFM image of Fig. 3b confirms that GO sheets are mostly few layers on the quartz substrate. After immersed in GO dispersion, the substrate with negatively charged GO sheets was then immersed into Au NPs colloid to fabricate the F-1 film. As depicted in Fig. 3c and the SEM image of Fig. 4a, it can be seen apparently that some Au NPs has been adsorbed onto the GO sheets. However, the dosage of Au NPs is sparse, which would limit the SERS enhancement effect of F-1 film. Obviously, the dosage of Au NPs on the F-2 film (in Fig. 3d and Fig. 4b) increases dramatically after two assembly cycles, which is significant for improving the SERS activity of the film. However, when the assembly cycles reach to three, not only the Au NPs in F-3 film (in Fig. 3e and Fig. 4c) have aggregated seriously, but also the GO layers has further increased, which might have negative influence on SERS property of the substrate.

Raman measurement is an efficient technique to characterize the carbonaceous materials such as graphene and its derivatives [1]. The Raman spectra (in Fig. 5a) of GO and Au NPs@GO films present the disorder $\mathrm{D}$ band at $1360 \mathrm{~cm}^{-1}$ and the tangential stretching mode ( $\mathrm{G}$ band) at $1590 \mathrm{~cm}^{-1}$ [25]. In order to further discuss the SERS activity of these films, R6G molecules were chosen as the probing molecules due to their distinctive Raman characteristic peaks and well adsorption ability. 
Fig. $5 \mathrm{~b}$ is the Raman spectra of R6G aqueous solution $(0.4 \mathrm{M})$ and the R6G molecules $\left(10^{-5} \mathrm{M}\right)$ adsorbed onto the GO film and F-2 film. Getting useful information from the Raman spectrum of pure R6G aqueous solution seems much difficult due to the high fluorescence of R6G molecules while the Raman intensity of R6G was enhanced visibly after adsorbed onto the Au NPs@GO films (in Fig. 5b, c), indicating the well fluorescence quenching property and distinct SERS enhancement of these films. To further evaluate the enhancement efficiency, the enhancement factor $(E F)$ values of these films have been calculated by quoting a simple formula: $E F=\left(I_{S E R S} / I_{\text {bulk }}\right) \times\left(N_{\text {bulk }} / N_{S E R S}\right)$, and the details are provided in the supporting information. Taking the typical band at $1650 \mathrm{~cm}^{-1}$ (the aromatic C-C stretching modes) for example, the $E F$ value is estimated to be $1.27 \times 10^{7}$ for GO film, $3.12 \times 10^{7}$ for pure Au NPs, $2.63 \times 10^{7}$ for F-1 film, $6.13 \times 10^{7}$ for F-2 film, and $5.94 \times 10^{7}$ for F-3 film, respectively. All of the calculated results are shown in Fig. 5d, in which the $E F$ values of the different substrates varied significantly. The $E F$ values of these films are about $10^{7}$, which is higher than many other studies published in the literature [26-28], but still lower than some works such as the published research reported by Leem et al. [29] and Yang et al. [5], indicating that our fabricated Au NPs@GO films have superior SERS activity. Meanwhile, the F-2 film presents the best SERS enhancement effect, which can be explained by the as-analyzed morphologies (in Fig. 3 and Fig. 4). In detail, the most Au NPs exposed on the surface of the F-2 film leads to close distance between the adjacent Au NPs, which can form "hot spots" (locations where the SERS enhancement is and the placement of the detected molecules within these regions) [30]. And the formed "hot spots" play a key role in improving the SERS performance against R6G molecules. However, due to the low dosage of Au NPs in the F-1 film and the increased GO layers [8] together with the relatively low Au NPs on the surface of the F-3 film, both of them cannot reach an optimal SERS enhancement. The above results indicate that the desirable SERS property of the Au NPs@GO films 
can be ascribed to the synergistic effects of the Au NPs as well as GO. Explicitly, the pure GO film has obvious SERS enhancement owing to the CM effect (in Fig. 5b) reported before [8]. The affluent oxygenous groups of GO together with large specific surface area render the Au NPs@GO films have excellent adsorbability against molecules, which is helpful for collecting the Raman signals. Meanwhile, the outstanding florescence quenching performance of GO can dramatically weaken the florescence of R6G molecules and therefore improve the SERS activity of the substrate. Moreover, due to the strong EM effect, Au NPs in the composite films dedicate the maximum SERS enhancement toward R6G molecules (in Fig. 5d). Thus, integrating the both enhancement effects of Au NPs and GO, the Au NPs@GO film presents the most excellent SERS property (in Fig. 5d) when compared with that of pure Au NPs and GO film.

Besides investigating the influence of assembly cycles on Au NPs@GO films, we also studied the surface compositions on SERS signals enhancement. Du et al. [31] took the CVD strategy to grow monolayer graphene, but the experimental condition of this method was harsh due to the CVD technology. Fan et al. [14] investigated the SERS property of Ag NPs@GO film at a single particle level, but the covered GO was mostly monolayer. Herein, the covered GO of our-prepared Au NPs@GO films was mostly few layers (in Fig. 3b). The two types of films: first with Au NPs on the surface (F-2 film in Fig. 3d and Fig. 4b) and the second with GO sheets (F-2-GO film in Fig. 3f and Fig. 4d) have been fabricated. As shown in Fig. 3d and Fig. 4b, there are a great number of Au NPs on the outer layer of the F-2 film. Differently, the surface of F-2-GO film is covered by few layers of GO sheets (in Fig. 3f and Fig. 4d). To demonstrate how the surface composition will influence the SERS signals of the two substrates, $1 \times 10^{-5} \mathrm{M}$ R6G molecules adsorbed on F-2 film and F-2-GO film were further investigated (Fig. 6b). Similarly, the F-2-GO film also gives an obvious improvement of the SERS intensities compared with R6G aqueous solution. But the SERS signal of 
F-2-GO film is weaker than that of the F-2 film shown in Fig. 6b. Interestingly, the result is the same as the typical Raman enhancement effect of these two films seeing from Fig. 6a, indicating the consistency of the Raman signals enhancement between typical Raman spectra and SERS spectra [24]. It was reported that a significant EM field enhancement can be obtained from a large-scale regularly arranged nanostructures with well-controlled narrow gaps (normally<10 nm), namely, "hot spots" [4]. Therefore, the EM field enhancement has its ranges of function. Camden et.al [32] reported that the SERS enhancement effect would decrease with the increase distance between "hot spots" and the detected molecules. As considered of our results, when the outer layer is covered with GO, the few-layer GO sheets would make the range between R6G molecules and "hot spots" become more distant which would make against the enhancement effect of Raman signals. However, when the surface is covered with Au NPs, the Raman signals will be enhanced significantly by EM effect. Also, when GO is under the R6G molecules, it can still give a CM enhancement by charge transfer between R6G molecules and GO sheets. Thus the synergetic effect of GO and Au NPs will reach to the optimum. On the basis of above discussions, the satisfactory SERS performance of the F-2 film can be attributed to the optimal synergetic effect of Au NPs and GO.

To investigate the reproducibility and stability of the as-prepared substrates, the F-2 film was chosen as an example. From 11 randomly chosen regions, the SERS curves of $1 \times 10^{-7} \mathrm{M}$ R6G molecules were firstly collected under certain test conditions. The relative standard deviation (RSD) curve was calculated according to the previous literature to reckon the reproducibility of SERS signals [33]. The superiority of this calculation method is that the RSD values of signal intensity of any Raman peak can be observed directly. The comparison results in Fig. 7 present that the mean RSD values of the whole Raman peaks are less than $18 \%$, which is better than that of the GO and gold nanorod hybrid material (smaller than 20\%) reported by Hu et al. [34], but is still inferior to 
that of the GO/Ag NPs hybrid material (below 15\%) referred by Liu et al. [15]. Furthermore, multiple samples (in Fig. S1) have been prepared to take the SERS spectra, which also present the superior reproducibility of the Au NPs@GO films. These results indicate that the uniform and reliable Au NPs@GO films with superior uniformity and reproducibility through the total areas have been synthesized successfully.

For the traditional SERS analyzing, the stability of the substrates is also meaningful. In order to evaluate this feature, the newly made substrate as well as the F-2 film reserved in air in excess of 2 months and 12 months were used for detecting R6G molecules. Almost no changes have been found from both the Raman intensity and characteristic peaks of R6G (in Fig.8), demonstrating that the as-mentioned Au NPs@GO films have high stability. The reason is due to the high stability of Au NPs itself as well as the high mechanical strength and adaptability of GO sheets, which can protect the substrates from destroying by the ambient environment.

\section{Conclusions}

In summary, an electrostatic self-assembly method for preparing the Au NPs@GO multilayer films with excellent SERS properties has been developed. The key role of assembly cycles in Au NPs@GO films has been investigated. Results indicate that the Au NPs@GO film with two assembly cycles exhibits the best SERS property for detecting R6G molecules contrasted with that of one assembly cycle and three assembly cycles due to the high Au NPs dosage and the formation of "hot spots" between the adjacent Au NPs. Meanwhile, the surface composition of the film was also investigated. It is found that the F-2 film with Au NPs on the surface has better SERS activity than the surface covered with GO sheets owing to the optimal synergetic effect of Au NPs and GO. Furthermore, the Au NPs@GO films also present favorable reproducibility and high stability, which 
are of great significance in the practical SERS applications.

Acknowledgements This work was supported by National Science Foundation of China (51572114), the Fundamental Research Funds for the Central Universities (No. 30920140122003), Senior Talent Foundation of Jiangsu University (15JDG078) and Students research project in Jiangsu University (14A335).

\section{References}

[1] X. Ling, S.X. Huang, S.B. Deng, N.N. Mao, J. Kong, M.S. Dresselhaus and J. Zhang, Lighting up the Raman signal of molecules in the vicinity of graphene related materials, Acc Chem. Res. 43 (7) (2015) 1862-1870.

[2] A. Hakonen, P.O. Andersson, M.S. Schmidt, T. Rindzevicius and M. Kall, Explosive and chemical threat detection by surface-enhanced Raman scattering: A review, Anal Chim Acta 893 (2015) 1-13.

[3] S.C. Luo, K. Sivashanmugan, J.D. Liao, C.K. Yao and H.C. Peng, Nanofabricated SERS-active substrates for single-molecule to virus detection in vitro: A review, Biosens Bioelectron. 61 (2014) 232-240.

[4] K.A. Willets, Super-resolution imaging of SERS hot spots, Chem. Soc. Rev. 43(11) (2014) 3854-3864.

[5] S Yang, X Dai, B.B. Stogin, T. Wong, Ultrasensitive surface-enhanced Raman scattering detection in common fluids, Proc. Natl. Acad. Sci. U. S. A. 113(2) (2016) 268-273.

[6]. C. Zhang, Y. Lu, B. Zhao, Y. Hao, Y. Liu, Facile fabrication of Ag dendrite-integrated anodic aluminum oxide membrane as effective three-dimensional SERS substrate, Appl. Surf. Sci. 377 
(2016) 167-173.

[7]. W. Xie, R. Grzeschik, S. Schlücker, Metal nanoparticle-catalyzed reduction using borohydride in aqueous media: a kinetic analysis of the surface reaction by microfluidic SERS, Angew. Chem. Int. Ed. 55 (2016) 1-6.

[8] X. Ling, L.M. Xie, Y. Fang, H. Xu, H.L. Zhang, J. Kong, M.S. Dresselhaus, J. Zhang and Z.F. Liu, Can Graphene be used as a Substrate for Raman Enhancement? Nano lett. 10 (2010) 553-561.

[9] M. Tabatabaei, M. Najiminaini, K. Davieau, B. Kaminska, M.R. Singh, J.J.L. Carson, F. Lagugné-Labarthet, Tunable 3D plasmonic cavity nanosensors for surface-enhanced Raman spectroscopy with sub-femtomolar limit of detection, ACS Photonics 2 (2015) 752-759.

[10] X.X. Yu, H.B. Cai, W.H. Zhang, X.J. Li, N. Pan, Y. Luo, X.P. Wang and J.G. Hou, Tuning chemical enhancement of SERS by controlling the chemical reduction of graphene oxide nanosheets, ACS Nano 5 (2011) 952-958.

[11] J. Hao, M. Han, S. Han, X. Meng, T. Su, Q.K. Wang, SERS detection of arsenic in water: A review, J. Environ. Sci. 36 (2015) 152-162.

[12] X.F. Ding, L.T. Kong, J. Wang, F. Fang, D.D. Li and J.H. Liu, Highly sensitive SERS detection of $\mathrm{Hg}^{2+}$, ACS Appl. Mater. Interfaces 5 (2013) 7072-7078.

[13] M. Iliut, C. Leordean, V. Canpean, C.M. Teodorescu and S. Astilean, A new green, ascorbic acid-assisted method for versatile synthesis of $\mathrm{Au}$-graphene hybrids as efficient surface-enhanced Raman scattering platforms, J. Mater. Chem C 1 (2013) 4094-4104.

[14] W. Fan, Y.H. Lee, S. Pedireddy, Q. Zhang, T.X. Liu and X.Y. Ling, Graphene oxide and shape-controlled silver nanoparticle hybrids for ultrasensitive single-particle surface-enhanced Raman scattering (SERS) sensing, Nanoscale 6 (2014) 4843-4851.

[15] X.J. Liu, L.Y. Cao, W. Song, K.L. Ai and L.H. Lu, Functionalizing metal nanostructured film 
with graphene oxide for ultrasensitive detection of aromatic molecules by surface-enhanced Raman spectroscopy, ACS Appl. Mater. Interfaces 3 (2011) 2944-2952.

[16] A. Shiohara, Y.S. Wang and L.M. Liz-Marzan, Recent approaches toward creation of hot spots for SERS detection, J. Photochem. Photobiol., C 21 (2014) 2-25.

[17] L. Zhang, L. Dai, Y. Rong, Z. Liu, D. Tong, Y. Huang, T. Chen, Light-triggered reversible self-assembly of gold nanoparticle oligomers for tunable SERS, Langmuir 31 (2015) 1164-1171.

[18] Y. Jia, S.N. Shmakov, E. Pinkhassik, Controlled permeability in porous polymer nanocapsules enabling size- and charge-selective SERS nanoprobes, ACS Appl. Mater. Interfaces 8 (2016) 19755-19763.

[19] S.K. Bhunia, L. Zeiri, J. Manna, S. Nandi, R. Jelinek, Carbon-dot/silver-nanoparticle flexible SERS-active films, ACS Appl. Mater. Interfaces 8 (2016) 25637-25643.

[20] Y. Li, J. Yang, Y.Z. Zhou, T. Zhong, S.H. Zheng, W.W. Zeng, Facile synthesis of gold nanoparticles-graphene oxide films and their excellent surface-enhanced Raman scattering activity, Monatsh Chem. 147 (2016) 677-683.

[21] H.J. Chen, X.S. Kou, Z. Yang, W.H. Ni and J.F. Wang, Shape- and size-dependent refractive index sensitivity of gold nanoparticles, Langmuir 24 (2008) 5233-5237.

[22] D.C. Marcano, D.V. Kosynkin, J.M. Berlin, A. Sinitskii, Z.Z. Sun, A. Slesarev, L.B. Alemany, W. Lu and J. M. Tour, Improved synthesis of graphene oxide, ACS Nano 4 (2010) 4806-4814.

[23] P.P. Zhang, Y. Huang, X. Lu, S.Y. Zhang, J.F. Li, G. Wei, Z.Q. Su, One-step synthesis of large-scale graphene film doped with gold nanoparticles at liquid-air interface for electrochemistry and Raman detection applications, Langmuir 30 (2014) 8980-8989.

[24] Y.Z. Zhou, X.N. Cheng, D. Du, J. Yang, N. Zhao, S.B. Ma, T. Zhong and Y.H. Lin, Graphenesilver nanohybrids for ultrasensitive surface enhanced Raman spectroscopy: size dependence of 
silver nanoparticles, J. Mater. Chem. C 2 (2014) 6850-6858.

[25] Y.S. Wang, L. Polavarapu and L.M. Liz-Marzan, Reduced graphene oxide-supported gold nanostars for improved SERS sensing and drug delivery, ACS Appl. Mater. Interfaces 6 (2014) 21798-21805.

[26] A.J. Caires, D.C.B. Alves, C. Fantini, A.S. Ferlauto, L.O. Ladeira, One-pot in situ photochemical synthesis of graphene oxide/gold nanorod nanocomposites for surface-enhanced Raman spectroscopy, RSC Adv. 5 (2015) 46552-46557.

[27] A. Saha, S. Palmal, N.R. Jana, Highly reproducible and sensitive surface-enhanced Raman scattering from colloidal plasmonic nanoparticle via stabilization of hot spots in graphene oxide liquid crystal, Nanoscale 4 (2012) 6649-6657.

[28] T.H.D. Nguyen, Z. Zhang, A. Mustapha, H. Li, M. Lin, Use of graphene and gold nanorods as substrates for the detection of pesticides by surface enhanced Raman spectroscopy, J. Agric. Food Chem. 62 (2014) 10445-10451.

[29] J. Leem, M.C. Wang, P. Kang, S. Nam, Mechanically self-assembled, three-dimensional graphene-gold hybrid nanostructures for advanced nanoplasmonic sensors, Nano Lett. 15 (2015) 7684-7690.

[30] G. Braun, L. Pavel, A.R. Morrill, D.S. Morrill, D.S. Seferos, G.C. Bazan, N.O. Reich and M. Moskovits, Chemically patterned microspheres for controlled nanoparticle assembly in the construction of SERS hot spots, J. Am. Chem. Soc. 129 (2007) 7760-7761.

[31] Y.X. Du, Y. Zhao, Y. Qu, C.H. Chen, C.M. Chen, C.H. Chuang and Y.W. Zhu, Enhanced lightmatter interaction of graphene-gold nanoparticle hybrid films for high-performance SERS detection, J. Mater. Chem. C 2 (2014) 4671-4683.

[32] J.P. Camden, J.A. Dieringer, Y.M. Wang, D.J. Masiello, L.D. Marks, G.C. Schatz and R.P. Van 
Duyne, Probing the structure of single-molecule surface-enhanced Raman scattering hot spots, J. Am. Chem. Soc. 130 (2008) 12617-12626.

[33] G. Mcnay, D. Eustace and W. E. Smith, Surface-enhanced Raman scattering (SERS) and surface-enhanced resonance Raman scattering (SERRS): A review of applications, Appl. Spectrosc. 65 (2011) 825-837.

[34] C. Hu, J. Rong, J. Cui, Y. Yang, L. Yang, Y. Wang, Y. Liu, Fabrication of a graphene oxidegold nanorod hybrid material by electrostatic self-assembly for surface-enhanced Raman scattering. Carbon 51 (2013) 255-264. 


\section{Figure captions:}

Fig. 1 The pictures of the obtained Au NPs: (a) UV-vis absorbance spectrum; (b) XRD pattern; (c) TEM image and (d) particle size distribution, the inserted picture is the Au colloid

Fig. 2 Preparation schematics of the Au NPs@GO film

Fig. 3 AFM images of (a, b) GO; (c) F-1 film; (d) F-2 film; (e) F-3 film and (f) F-2-GO film

Fig. 4 SEM images of (a) F-1 film; (b) F-2 film; (c) F-3 film and (d) F-2-GO film

Fig. 5 (a) Typical Raman spectra of GO, F-1 film, F-2 film and F-3 film; SERS spectra of $1 \times 10^{-5} \mathrm{M}$ R6G molecules absorbed on different substrates (in (b) and (c)), for comparison, Raman spectra of 0.4 M R6G aqueous solution is presented; (d) the $E F$ values of different substrates of Raman shift at band of 612,1380 and $1650 \mathrm{~cm}^{-1}$

Fig. 6 (a) Typical Raman spectra of F-2 and F-2-GO film; (b) SERS spectra of $1 \times 10^{-5}$ M R6G molecules absorbed on F-2 film and F-2-GO film

Fig. 7 Picture of RSD-SERS: SERS curves of R6G $\left(1 \times 10^{-7} \mathrm{M}\right)$ gathered from 11 random regions of the film (upper) and the relevant RSD values curve (down)

Fig. 8 SERS spectra of R6G molecules adsorbed on F-2 film obtained from the substrates (a) the newly made; (b) in excess of 2 months; and (c) over 12 months 
Figure 1


Fig. 1 The pictures of the obtained Au NPs: (a) UV-vis absorbance spectrum; (b) XRD pattern; (c) TEM image and (d) particle size distribution, the inserted picture is the Au colloid

\section{Figure 2}

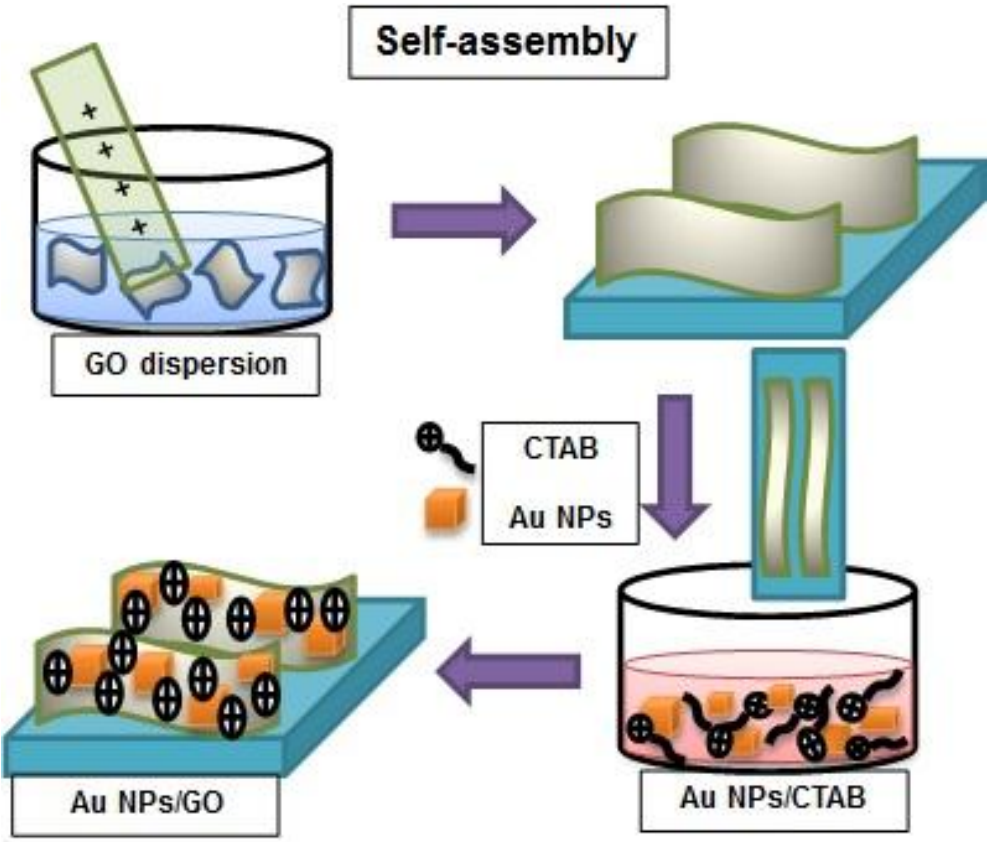

Fig. 2 Preparation schematics of the Au NPs@GO film 


\section{Figure 3}
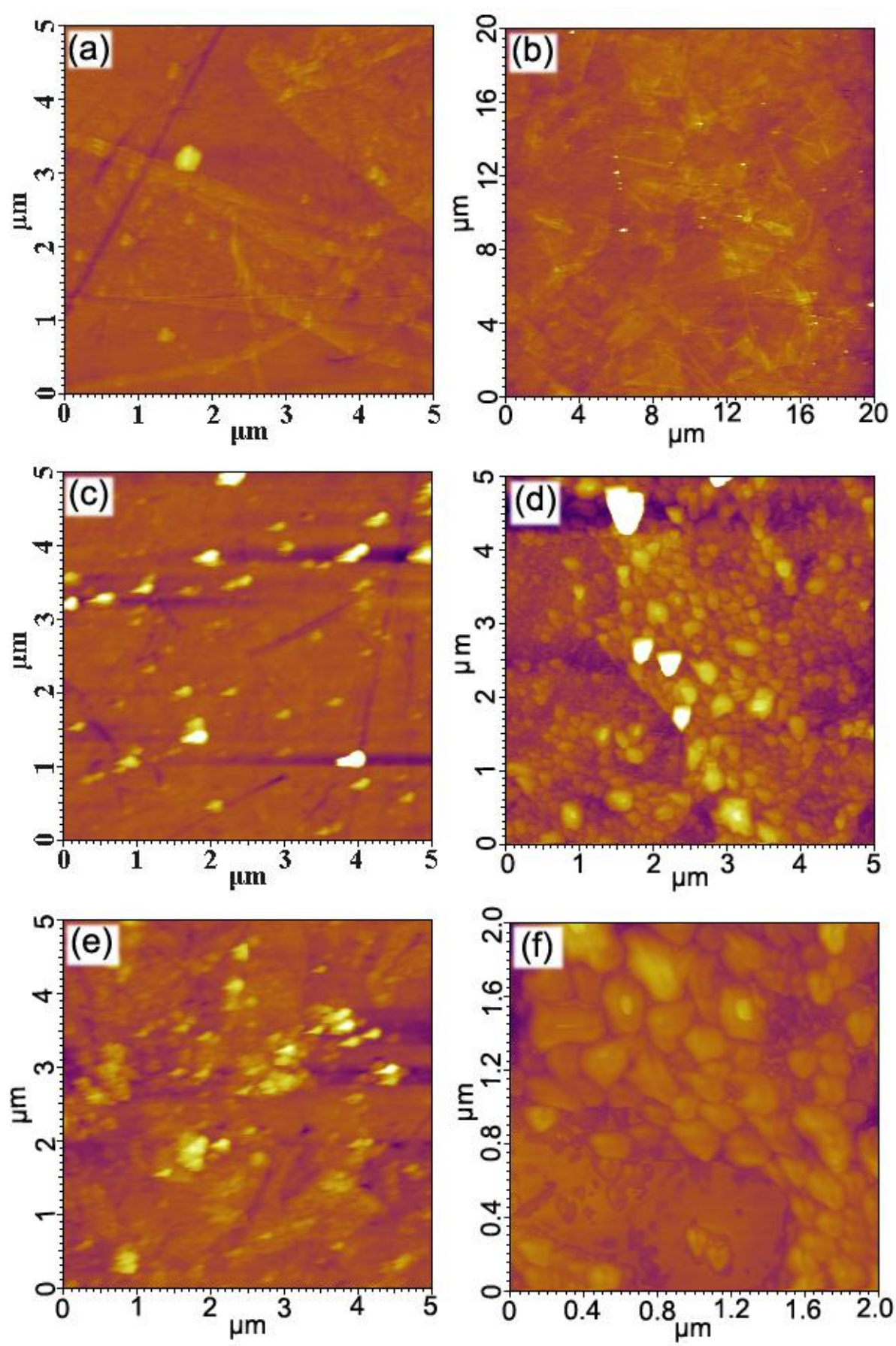

Fig. 3 AFM images of (a, b) GO; (c) F-1 film; (d) F-2 film; (e) F-3 film and (f) F-2-GO 


\section{Figure 4}
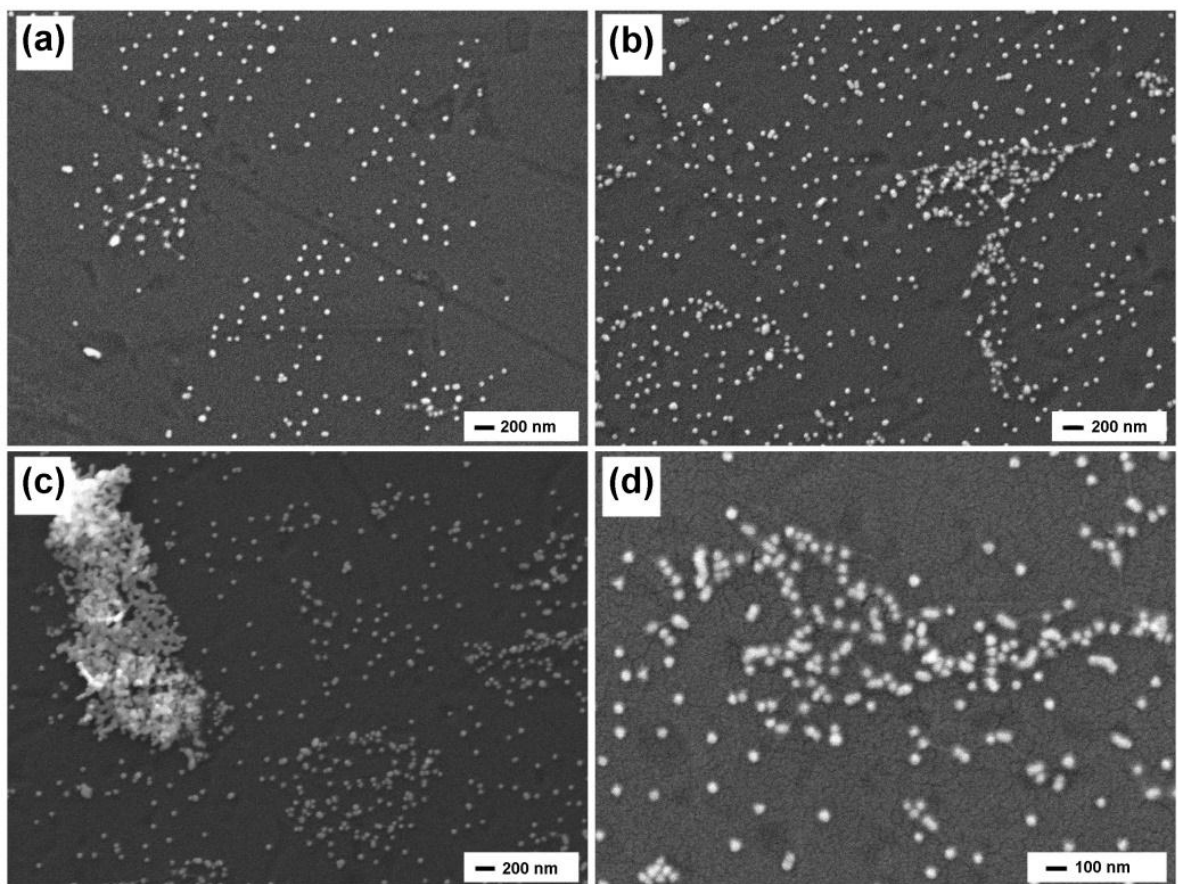

Fig. 4 SEM images of (a) F-1 film; (b) F-2 film; (c) F-3 film and (d) F-2-GO film

\section{Figure 5}
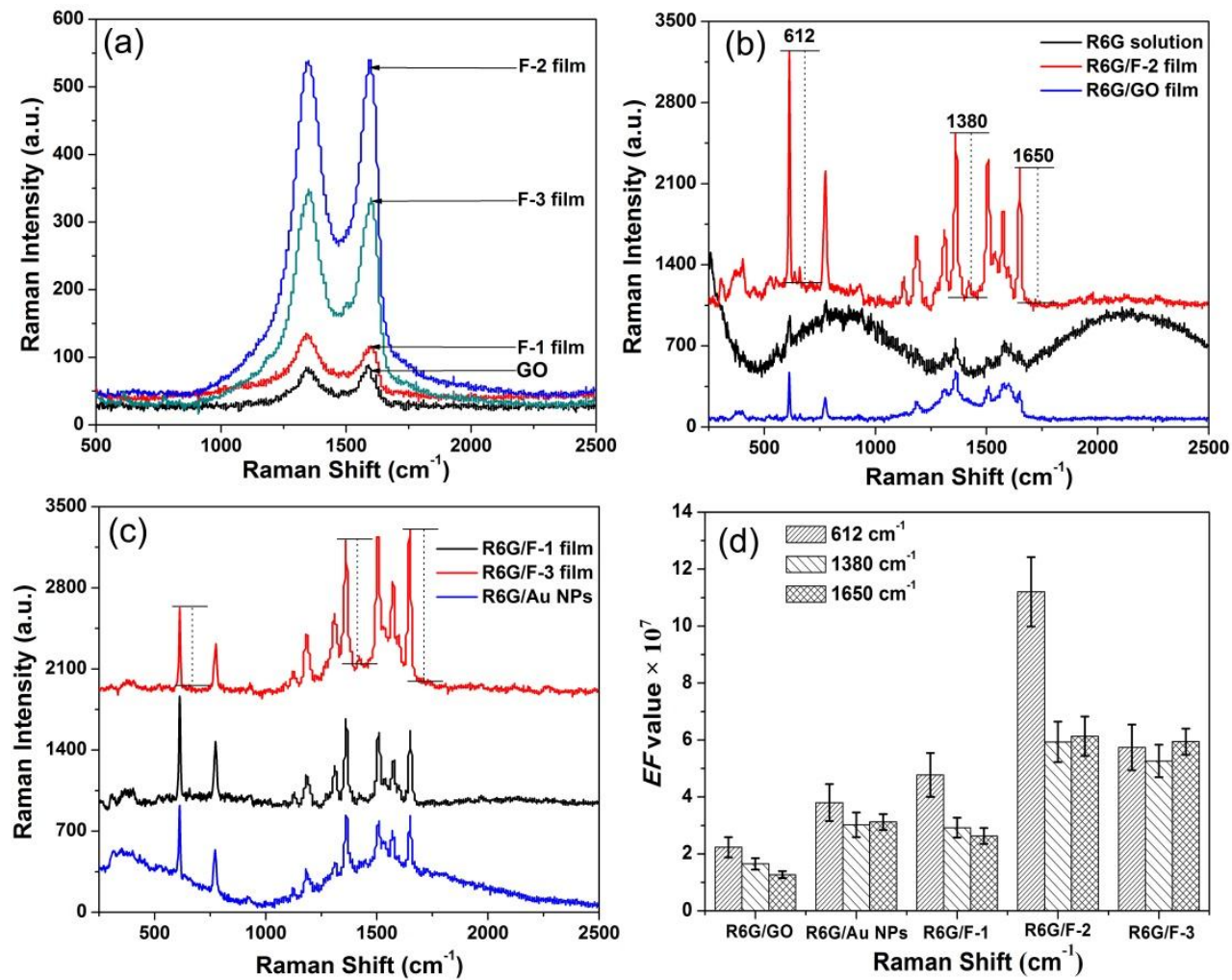

Fig. 5 (a) Typical Raman spectra of GO, F-1 film, F-2 film and F-3 film; SERS spectra of $1 \times 10^{-5} \mathrm{M}$ R6G molecules absorbed on different substrates (in (b) and (c)), for comparison, Raman spectra of 0.4 M R6G aqueous solution is presented; (d) the $E F$ values of different substrates of Raman shift at band of 612,1380 and $1650 \mathrm{~cm}^{-1}$ 


\section{Figure 6}
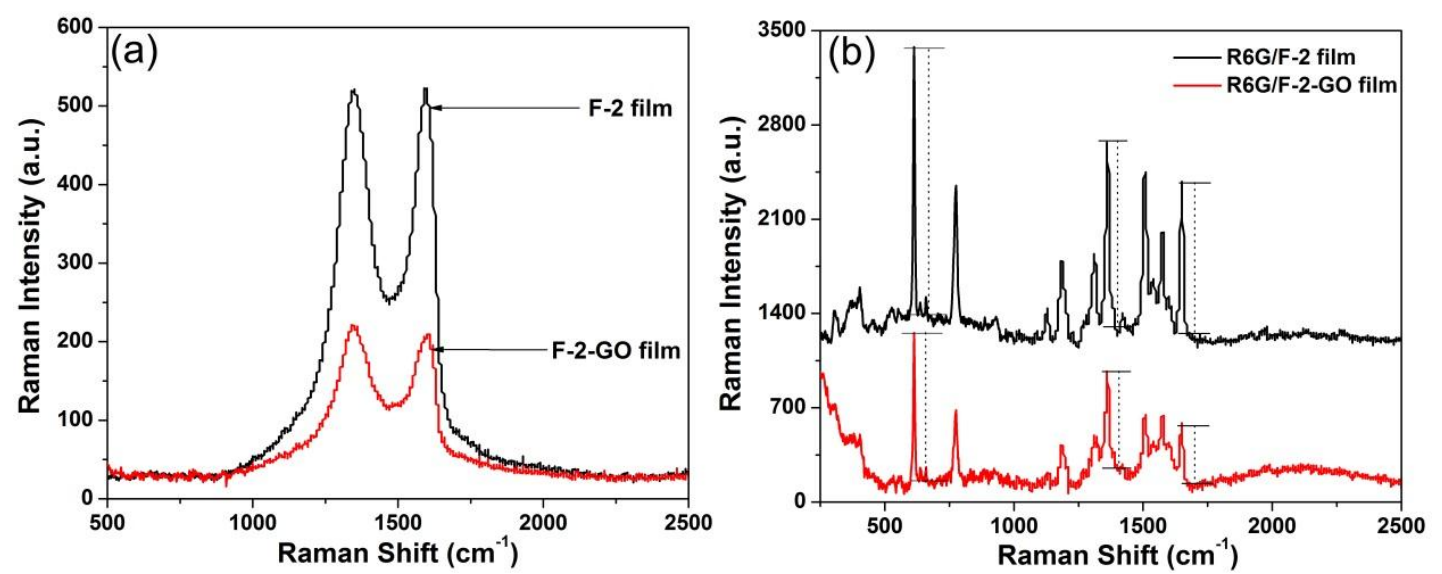

Fig. 6 (a) Typical Raman spectra of F-2 and F-2-GO film; (b) SERS spectra of $1 \times 10^{-5}$ M R6G molecules absorbed on F-2 film and F-2-GO film

\section{Figure 7}

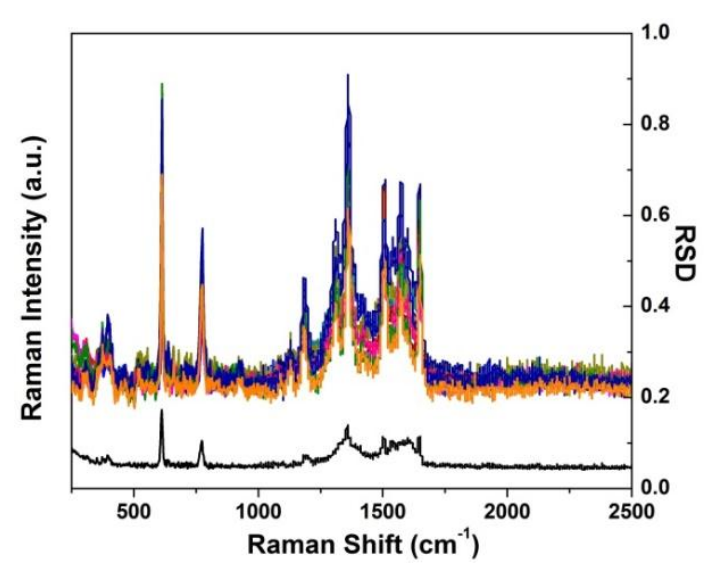

Fig. 7 Picture of RSD-SERS: SERS curves of R6G $\left(1 \times 10^{-7} \mathrm{M}\right)$ gathered from 11 random regions of the film (upper) and the relevant RSD values curve (down)

Figure 8

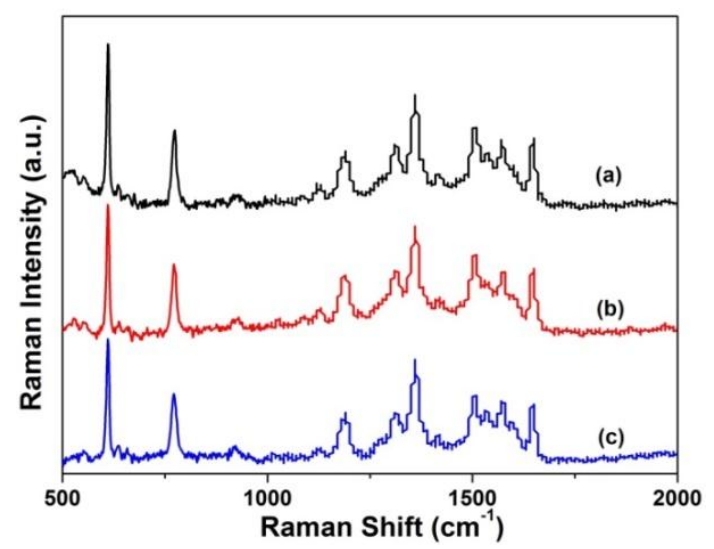

Fig. 8 SERS spectra of R6G molecules adsorbed on F-2 film obtained from the substrates (a) the newly made; (b) in excess of 2 months; and (c) over 12 months 
Graphical abstract:

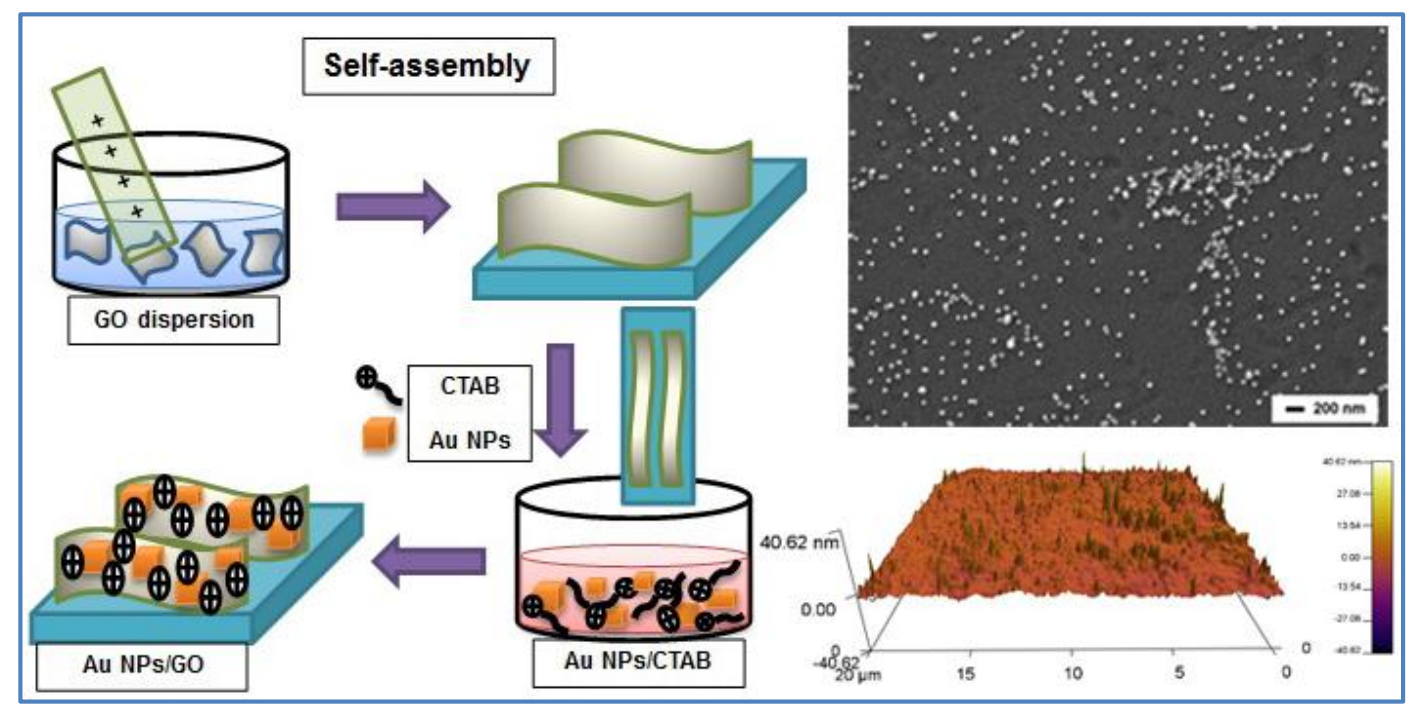

